\title{
Implications for Systemic Approaches to COVID-19: Effect Sizes of Remdesivir, Tocilizumab, Melatonin, Vitamin D3, and Meditation
}

\author{
Ryan D Castle $\mathbb{D}^{\prime}$ \\ Michelle A Williams ${ }^{2}$ \\ William C Bushell ${ }^{3}$ \\ J Adam Rindfleisch ${ }^{4}$ \\ Christine Tara Peterson ${ }^{5}$ \\ James Marzolf $\mathbb{D D}^{6}$ \\ Kimberly Brouwer ${ }^{7}$ \\ Paul J Mills ${ }^{8}$
}

\begin{abstract}
'Science Division, Whole Health Institute, Bentonville, AR, USA; ${ }^{2}$ Harvard T.H. Chan School of Public Health, Department of Global Health and Population, Harvard University, Boston, MA, USA; ${ }^{3}$ Science Division, Whole Health Institute, New York, NY, USA;

${ }^{4}$ Education Department, Whole Health School of Medicine and Health Sciences, Bentonville, AR, USA; ${ }^{5}$ Center of Excellence for Research and Training in Integrative Health, Department of Family Medicine, School of Medicine, University of California, San Diego, CA, USA; ${ }^{6}$ Health Sector Finance \& Policy, Whole Health Institute, Bentonville, AR, USA; ${ }^{7}$ Herbert Wertheim School of Public Health and Human Longevity Science, University of California, San Diego, CA, USA; ${ }^{8}$ Herbert Wertheim School of Public Health and Human Longevity Science, Center of Excellence for Research and Training in Integrative Health, University of California, San Diego, CA, USA
\end{abstract}

Correspondence: Ryan D Castle Science Division, Whole Health Institute, 900 McClain Road, Bentonville, AR, 727I2, USA

Email rcastle@wholehealth.org
Introduction: COVID-19 poses a chronic threat to inflammatory systems, reinforcing the need for efficient anti-inflammatory strategies. The purpose of this review and analysis was to determine the efficacy of various interventions upon the inflammatory markers most affected by COVID-19. The focus was on the markers associated with COVID-19, not the etiology of the virus itself.

Methods: Based on 27 reviewed papers, information was extracted on the effects of COVID-19 upon inflammatory markers, then the effects of standard treatments (Remdesivir, Tocilizumab) and adjunctive interventions (vitamin $\mathrm{D}_{3}$, melatonin, and meditation) were extracted for those markers. These data were used to approximate effect sizes for the disease or interventions via standardized mean differences (SMD).

Results: The data that were available indicated that adjunctive interventions affected $68.4 \%$ of the inflammatory markers impacted by COVID-19, while standard pharmaceutical medication affected $26.3 \%$.

Discussion: Nonstandard adjunctive care appeared to have comparable or superior effects in comparison to Remdesivir and Tocilizumab on the inflammatory markers most impacted by COVID-19. Alongside standards of care, melatonin, vitamin $\mathrm{D}_{3}$, and meditation should be considered for treatment of SARS-COV-2 infection and COVID-19 disease.

Keywords: COVID-19, inflammation, cytokine storm, effect size, antivirals, corticosteroids, melatonin, vitamin $\mathrm{D}$, meditation, whole health

\section{Introduction}

The COVID-19 pandemic has had pervasive adverse consequences, especially in the United States. ${ }^{1}$ The combination of a highly contagious virus with significant hospitalization and mortality rates has resulted in devastating loss of human life and social stability. These costs continue to increase as research uncovers wider and more serious consequences for those who survive the initial infection, ranging from autoimmune disorders to fatal cardiopulmonary events. ${ }^{2}$ The diverse and unpredictable nature of these complications point to a disease that is more complex than a respiratory virus. ${ }^{3}$ It is increasingly apparent that SARS-CoV-2 infection can lead to an inflammatory disorder affecting numerous bodily systems, with lasting effects on systemic inflammation that will linger long after the virus is eliminated.

Evidence suggests that approaching this systemic disease with systemic adjunctive interventions such as vitamin $\mathrm{D}_{3}$, melatonin, and meditation could prove more effective than targeting acute symptoms solely with pharmacological means such as 
Remdesivir and Tocilizumab. ${ }^{4-6}$ The purpose of this work is to track the effects of various catalysts, including Remdesivir, Tocilizumab, melatonin, meditation, vitamin $\mathrm{D}_{3}$, and COVID-19 itself, on a variety of inflammatory markers. The initial hypothesis explores whether adjunct interventions show any significant effects on inflammatory markers that Remdesivir and Tocilizumab do not affect. If so, that would imply those interventions may be viable complement in the treatment of COVID-19.

\section{Inflammatory Model of Disease}

A significant body of evidence suggests that acute and chronic inflammation accompanies and underlies many noncommunicable diseases. ${ }^{7}$ Inflammation is critical to a healthy host's defense against infection and injury, however, when continually activated it contributes to the ongoing pathophysiology of many chronic diseases. A persistent, low-level inflammatory response affects the innate immune system, adaptive immune system, and inflammatory mediators. ${ }^{8}$ The dynamic mechanisms of chronic inflammation can lead to tissue damage, oxidative stress, angiogenesis, and fibrosis in a variety of tissues, including the nervous system. ${ }^{9}$

Many of the most damaging effects of COVID-19 are related to cascading systemic inflammation and resulting cytokine storms, affecting respiratory, cardiac, nervous, gastrointestinal, and immune systems concurrently. ${ }^{10,11}$ Patterns of comorbidity demographics indicate that COVID-19 both initiates inflammatory dysregulation and exacerbates existing systemic inflammation. ${ }^{12}$ The prevalence and magnitude of stressors contributing to preexisting inflammation make a catalyst like COVID-19 especially dangerous. ${ }^{13}$

\section{COVID Reveals Lingering Vulnerability}

Though vaccines have been developed to eventually curb the spread of the virus itself, there are already more than 135 million infected so far, a number that is expected to rise for months. ${ }^{14}$ It is important to note that the elimination of the virus, whether in an individual host or in a population, does not eliminate the increased health risks. Some individuals who have fully cleared the virus still exhibit significantly impaired cardiopulmonary, central nervous, and peripheral nervous systems. ${ }^{15}$ This suggests an eventual potential spike in pulmonary fibrosis, cardiopulmonary events, neuronal damage, Alzheimer's disease, Parkinson's disease, and multiple sclerosis. ${ }^{16-18}$
In addition to direct health impacts, the COVID-19 pandemic has highlighted the vulnerabilities of healthcare systems. The heavy load on hospitals was likely exacerbated by high rates of metabolic syndrome, a low-grade inflammatory condition that is suspected to afflict $10-40 \%$ of the developed world's population and correlates to COVID-19 severity and comorbidities. ${ }^{19}$ Lengthy recovery times and high prevalence of comorbidity resulted in overcrowded hospitals with rates of readmission as high as $1 / 6 .^{20}$ If COVID-19 were to mutate enough to escape the vaccines or if a new inflammatory pathogen emerges, the population is currently even more vulnerable than at the beginning of the current pandemic, with few effective mitigating interventions.

Ending the spread of COVID-19 will not address the health needs of the survivors, nor erase the conditions that exacerbated the pandemic. In fact, authorities have predicted that the long-term public health impact of COVID19 will be profound; and in December 2020, Congress provided $\$ 1.15$ billion in funding for the National Institutes of Health to support research into the prolonged health consequences of SARS-CoV-2 infections. ${ }^{21}$

\section{Mechanism of Action: COVID-19}

While the inflammatory reaction and release of cytokines in healthy individuals is a necessary and normal reaction to injury and infection, in the case of inflammatory diseases cascading overproduction of cytokines can occur. COVID-19 often dysregulates pro-inflammatory chemokines that continue to stimulate this reaction until multiorgan damage occurs, often with fatal consequences for the infected individual. ${ }^{22}$ The mechanism suspected in severe occurrences of the SARS-CoV2 virus is the cytokine storm, an event involving hyperactivity of immune cells that produce pro-inflammatory cytokines, referred to as hypercytokinemia, which can lead to severe or even life-threatening cases of multiple organ failures. ${ }^{23}$

Even though inflammatory symptoms may be more visible in particular parts of the body, the nature of cytokine storms is systemic, not acute or localized. Once chemokine and cytokine interactions have begun to spiral out of homeostasis, a system-wide feedback loop can be established which triggers effects in an extremely diverse set of bodily functions and organs. ${ }^{24}$ Pulmonary complications, blood clots, and neurological issues are closely interwoven with systemic inflammation, making treatment of individual symptoms difficult. ${ }^{25}$

Elevated TGF-beta plasma levels have been observed in respiratory diseases such as COPD and COVID-19 and 
maintain a central role in the pathophysiology of lung fibrosis during inflammation. ${ }^{26,27}$ In COVID-19 patients, TGF-beta upregulation contributes to cytokine release syndrome, pulmonary edema and acute respiratory distress syndrome via increased capillary permeability. ${ }^{28-31}$ The complement system further drives TGF-beta release from platelets to promote systemic inflammation and coagulopathy. ${ }^{32}$ SARS-CoV-2 infections also promote increased MAPK pathway mediated inflammation. ${ }^{33,34}$
Figure 1 was created by this research team to outline the cellular and systemic progression of COVID-19 and the inflammatory response:

\section{Mechanism of Action: Systemic Adjunct Interventions}

The type of systemic dysregulation that both fuels the worst cases of COVID-19 and poses a lingering risk has been the subject of significant research relating to the

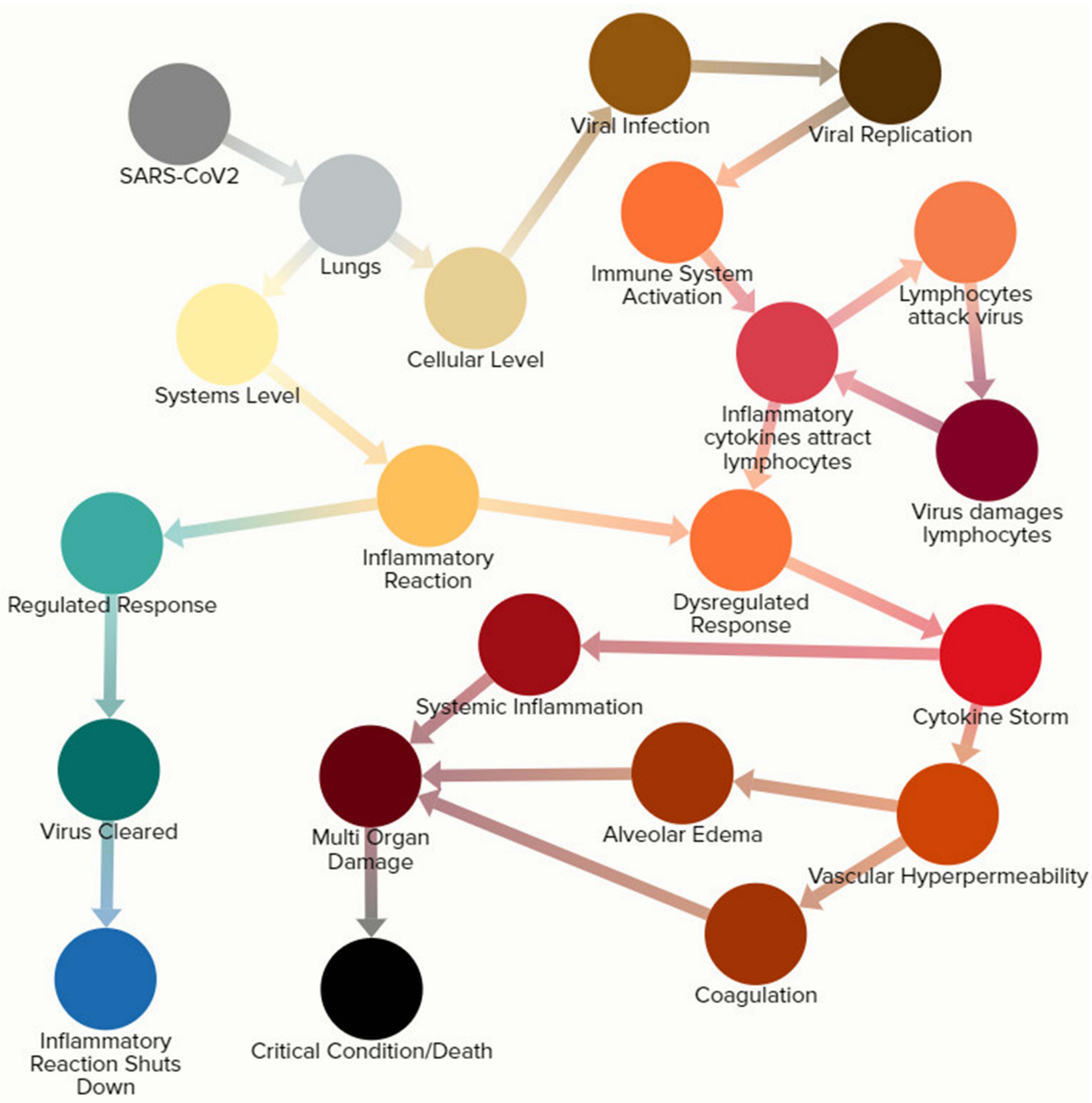

Figure I Progression and feedback loops associated with cytokine storms.

Note: Courtesy of the Whole Health Institute. 
efficacy of non-pharmacological adjunct interventions. ${ }^{35}$ A particular area of focus has been referred to as "integrative medicine," which incorporates systems-level approaches to health that include behavioral changes, mental practices, and nutritional adjustments, interventions which have been associated with significant improvements in stress levels, pain, cardiovascular health, and metabolic health. ${ }^{36}$ Examples of these adjunct interventions that are relevant to COVID-19 treatment include meditation, vita$\min \mathrm{D}_{3}$ (cholecalciferol), and melatonin. ${ }^{37-39}$

Targeted modulation of key cytokines such as TGF-beta with natural products such as artemisinin, an antimalarial lactone derived from Artemisia annua, may potentially help prevent pulmonary fibrosis in patients with respiratory diseases such as COVID-19 and could act as a potent cytokine-modulating adjunctive therapy as well, but insufficient data was present to include artemisinin or TGF-beta in this analysis. $^{40-43}$

The mechanisms of action for these interventions are still being studied, but existing evidence suggests regulating influences that have significant effects on dysregulated inflammatory processes. Vitamin $\mathrm{D}_{3}$, for example, has been shown to improve immune function while also curbing cascading inflammation, with numerous studies linking it to reduced severity and deaths in COVID-19 cases. ${ }^{44-46}$ The mechanism(s) behind melatonin is slightly less understood but potentially has the intriguing benefit of upregulating antiviral function while downregulating runaway inflammation. ${ }^{47}$

Meditation has been linked to diverse psychological and physiological changes that improve homeostasis and diminish inflammatory feedback loops, though its mechanisms are still widely debated. ${ }^{48}$ Theoretical modeling suggests behavioral health practices such as meditation and yoga may provide some degree of adjunctive efficacy against general infectious conditions through enhancements of endogenous levels of melatonin and other antiinflammatory or immuno-modulating processes. ${ }^{49}$ This model was expanded to directly relate to COVID-19, with multidisciplinary evidence supporting the premise.

None of these adjunct interventions have been linked to any dangerous side effects during normal administration. It is important to note that the efficacy of an adjunct treatment does not replace or disprove standards of care. Such interventions are intended to supplement medical best practices, not replace them.

\section{Central Role of Systems Approach}

The systemic nature of cascading inflammation makes it difficult to treat with interventions that are more targeted in nature. Many of the pharmacological interventions that are capable of significant system-wide effects, such as the leading COVID-19 medications Remdesivir and Tocilizumab, are often accompanied by negative sideeffects. ${ }^{50,51}$ Therefore, it is imperative to establish adjunctive interventions that can address the broader, holistic factors behind COVID-19 and do not result in dangerous side effects or numerous contraindications.

While a reductionist model is ideal for acute conditions, COVID-19 and its complications require a systems model that incorporates multiple levels of connection, causality, and interaction. There is insufficient data to calculate a full systems analysis of COVID-19 and potential interventions, but a mapping of the effects of the disease and its treatments is an important step toward understanding the most effective way to address this pandemic and any others like it. At the time of the writing of this research no such systems model for COVID-19 is known.

\section{Methodology}

The purpose of this article is to conduct a review and comparative analysis of the effect sizes of COVID-19, Remdesivir, Tocilizumab, meditation, vitamin $\mathrm{D}_{3}$, and melatonin on a variety of inflammatory markers.

\section{Data}

Figure 2 reflects the compilation process for this research, through which a collection of search terms was designed to capture a wide sampling of sources related to quantitative interactions between inflammatory processes/markers and COVID-19, Remdesivir, Tocilizumab, melatonin, meditation, and vitamin $\mathrm{D}_{3}$. Remdesivir and Tocilizumab were selected for analysis as a result of being the predominant pharmacological intervention for COVID at the time of data-gathering. Vitamin $\mathrm{D}_{3}$ and melatonin were included as adjunctive interventions due to their repeated use in COVID infections, while meditation was also included as an adjunct intervention to analyze a potentially anti-inflammatory intervention that did not introduce external properties to the subject. ${ }^{52}$ Though there are multiple meditative practices, the meditation studies included in this review focused primarily on mindfulness meditation. 


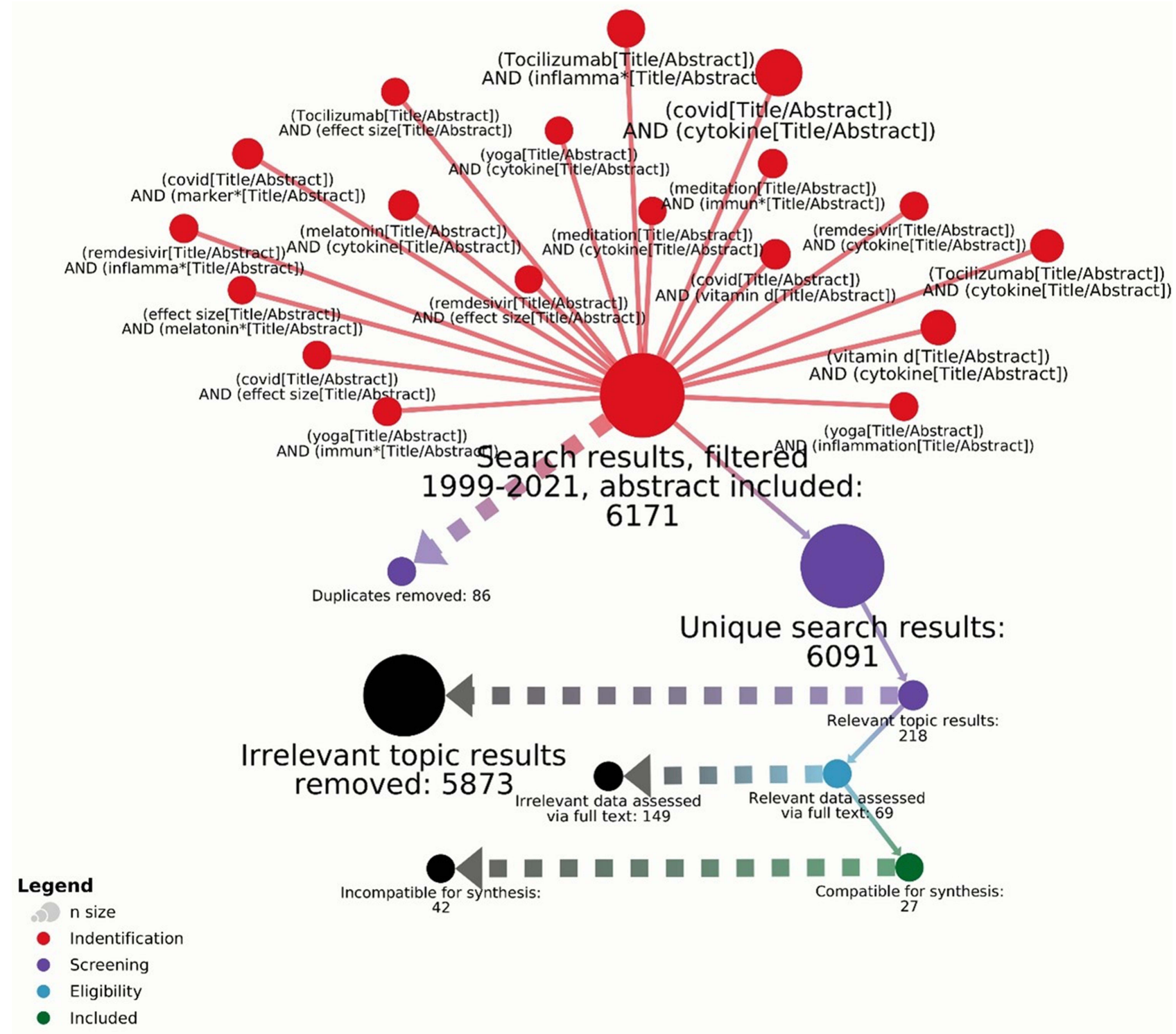

Figure 2 Outline of the source selection process for this review. The process was relatively heterogeneous but primarily focused on adult populations across the world.

Due to the extremely recent nature of COVID-19 and the lack of significant research into many adjunctive approaches, selection also focused on known effects on biomarkers associated with SARS-CoV2 infection, not only on effects specific to COVID-19 infection. The markers tracked in interventions was moderately heterogeneous across studies and availability was a limiting factor in which interventions could be effectively tracked. The search terms were filtered to include studies providing an abstract and published 1999-2021, the date range being intended to ensure current terminology and comprehension of inflammatory disorders. Table 1 lists the search terms developed internally for this study:
Searches were conducted primarily through PubMed and subjected to topical identification, screening for relevance, full-text reading for practically relevant data, and included if all of the above allowed coherent synthesis and analysis. Metrics that were compiled in systematic reviews were compared to the original publication to ensure fidelity.

The final results for review and analysis include: Aiswarya et al, ${ }^{53}$ 2021; Akbari et al, 2020; ${ }^{54}$ Alamdari et al, 2015; ${ }^{55}$ Bartels et al, 2013; ${ }^{56}$ Bower et al, 2015; ${ }^{57}$ Cahn et al, 2017; ${ }^{48}$ Carlson et al, 2003, ${ }^{58} 2004,{ }^{59} 2007 ; ;^{60}$ Celinski et al, 2014; ${ }^{61}$ Chen et al, 2020; ${ }^{62}$ L. Chen et al, $2020 ;{ }^{63}$ Cichoz-Lach et al, 2010; ${ }^{64}$ Epel et al, 2016; ${ }^{65}$ 
Table I Search Terms Included in Selection of Sources for Review and Analysis

\begin{tabular}{|l|}
\hline Search Terms \\
\hline (CovidTitle/Abstract) AND (effect sizeTitle/Abstract) \\
\hline (Effect sizeTitle/Abstract) AND (melatonin*Title/Abstract) \\
\hline (CovidTitle/Abstract) AND (vitamin dTitle/Abstract) \\
\hline (RemdesivirTitle/Abstract) AND (cytokineTitle/Abstract) \\
\hline (TocilizumabTitle/Abstract) AND (cytokineTitle/Abstract) \\
\hline (RemdesivirTitle/Abstract) AND (effect sizeTitle/Abstract) \\
\hline (TocilizumabTitle/Abstract) AND (effect sizeTitle/Abstract) \\
\hline (RemdesivirTitle/Abstract) AND (inflamma*Title/Abstract) \\
\hline (TocilizumabTitle/Abstract) AND (inflamma*Title/Abstract) \\
\hline (CovidTitle/Abstract) AND (cytokineTitle/Abstract) \\
\hline (MeditationTitle/Abstract) AND (cytokineTitle/Abstract) \\
\hline (MeditationTitle/Abstract) AND (immun*Title/Abstract) \\
\hline (CovidTitle/Abstract) AND (marker*Title/Abstract) \\
\hline (YogaTitle/Abstract) AND (inflamma*Title/Abstract) \\
\hline (YogaTitle/Abstract) AND (cytokineTitle/Abstract) \\
\hline (YogaTitle/Abstract) AND (immun*Title/Abstract) \\
\hline (MelatoninTitle/Abstract) AND (cytokineTitle/Abstract) \\
\hline (Vitamin dTitle/Abstract) AND (cytokineTitle/Abstract) \\
\hline
\end{tabular}

Gonzalez-Garcia et al, 2014; ${ }^{66}$ Javanmard et al, 2016; ${ }^{67}$ Lengacher et al, 2013; ${ }^{68}$ Qin et al, 2020a, ${ }^{69} 2020 \mathrm{~b} ;{ }^{69}$ Rausch-Fan et al, 2002; ${ }^{70}$ Raygan et al, 2019; ${ }^{71}$ Rosenkranz et al, 2016; ${ }^{72}$ Shete et al, 2017; ${ }^{73}$ Smolen et al, 2008; ${ }^{74}$ Wang et al, 2020 ${ }^{75}$ Witek-Janusek et al, $2008 ;^{76}$ Zhang et al, $2020 .^{39}$

\section{Biomarker Selection}

Disease markers were selected for inclusion if there were sufficient empirical evidence from research to calculate COVID-19 effects (Table 2). ${ }^{39,48,53-76}$ Intervention effects were included if they affected at least one inflammatory marker.

\section{Exceptions to This Rule Include CD3}

Though there were insufficient data to estimate effect sizes with COVID-19 status, there was evidence of close associations between CD3 and COVID-19's inflammatory effects. ${ }^{77}$
Table 2 Markers, Largely Inflammatory, Selected from Those Most Impacted by COVID-19

\begin{tabular}{|c|c|}
\hline \multicolumn{2}{|l|}{ Markers } \\
\hline CDI9 & IL-6 \\
\hline CD3 & IL-6 classic \\
\hline CD4 & IL- 6 trans \\
\hline CD8 & IL-8 \\
\hline Cortisol & INF- $\lambda$ \\
\hline CRP & Lymphocytes \\
\hline Eosinophil & Monocyte \\
\hline ESR (erythrocyte sedimentation rate) & NK cell (natural killer cell) \\
\hline Ferritin & Th Memory cells \\
\hline IL-10 & Th Naïve cells \\
\hline IL-2 & TNF a \\
\hline IL-4 & \\
\hline
\end{tabular}

\section{Cortisol}

Cortisol was widely recorded as a predictive marker of severe COVID-19 cases and was tracked with sufficient data across several interventions, so this marker was included despite insufficient data to calculate COVID-19

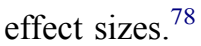

\section{IL-6}

There were ample data among both COVID-19 research and the various interventions to calculate effects on interleukin 6 , but a specific distinction deserves note. IL-6 is often reported as a pro-inflammatory marker and is one of the most dysregulated cytokines affected by COVID-19, but there have also been interactions with IL-6 that were wildly inconsistent across studies, sometimes demonstrating behavior directly opposing what is expected of an inflammatory marker. ${ }^{28,56}$ A possible explanation involves research indicating that there are two forms of IL-6, a proinflammatory IL-6 trans and a regulating antiinflammatory IL-6 classic. ${ }^{79}$ All effects on IL-6 were calculated and regulating effects categorized as IL-6 classic, while dysregulating effects were categorized as IL-6 trans. Note that this is an internal categorization and does not reflect differentiation in the data. Should the premise of two forms of IL- 6 be discounted the effect sizes toward a combined IL-6 will not be affected. 


\section{Effect Size vs Significance}

Effect size by standardized mean difference (SMD or $d$ ) differs from statistical significance $(p)$ and is the primary metric explored in this paper. This is driven by the fact that significance reports how likely a difference is due to chance, while effect size reports how large the difference is. When attempting to compare the potential effects of diverse interventions, $p$ alone is insufficient to determine efficacy. The need for effect sizes when examining tangible effects was explained by the American Statistical Association:

Statistical significance is not equivalent to scientific, human, or economic significance. Smaller p-values do not necessarily imply the presence of larger or more important effects, and larger p-values do not imply a lack of importance or even lack of effect. ${ }^{80}$

The majority of sources used in this analysis did not provide effect sizes. When sources did not include a reported standardized mean difference, an effect size $(d)$ was calculated using test mean $(M)$, control mean, and pooled standard deviations $(S D)$. When sources did not include standard deviations, SD was calculated using sample size (n), and a minimum/maximum range of reported values. When sources only included weighted mean difference, these were converted to standardized mean difference using the WMD value, $\mathrm{n}$, the minimum/ maximum range of reported values, and the SD. If the above data were not available the study was designated as incompatible for synthesis and excluded.

$$
\begin{gathered}
d=\left(M_{2}-M_{1}\right) / S D_{\text {pooled }} \\
S D_{\text {pooled }}=\sqrt{ }\left(\left(S D_{1}{ }^{2}+S D_{2}{ }^{2}\right) / 2\right)
\end{gathered}
$$

Where SMD were categorized the size guidelines of Cohen's $d$ were followed with some adjustments: ${ }^{81}$

- Below 0.1 is negligible effect size.

- $0.1-0.2$ is very small effect size.

- $0.2-0.5$ is small effect size.

- $0.5-0.8$ is medium effect size.

- $0.8-1.0$ is large effect size.

- Above 1.0 is very large effect size.

Though extensive effort was made to ensure accuracy, due to the diversity of metrics and conversion to different measurement systems, all SMD effect sizes reported in this analysis should be considered approximate.

\section{System Analysis}

This analysis is a preliminary step toward a systems-level examination of COVID-19 effects and interventions. A systemic disease requires a systemic research approach, which itself requires a comprehension of a wide array of interconnections. Though the scope of this initial paper does not include a complex systems model, multiple network analyses were performed. These metrics will inform not only the effects of individual interventions, but the density of overlap between the effects of the disease and interventions in connection with different inflammatory markers.

\section{Results}

Given the complex nature of the immune system and cytokines in particular, simple categorization is problematic, but broad generalities have been applied. Markers whose increase is usually indicative of dysregulated inflammation have been labelled as inflammatory markers, while markers whose increase is usually indicative of a healthy, regulated response are labelled as antiinflammatory markers. Catalyst effects which increase anti-inflammatory markers are labelled as regulating while catalyst effects which increase inflammatory markers are labelled as dysregulating. These are only broad tendencies and shall not be interpreted as universal qualities nor precise descriptions of the nuanced behavior of these markers.

The studies incorporated into this analysis included numerous severity levels of COVID-19, different dosages of Remdesivir and Tocilizumab, and various durations of adjunctive interventions. A limiting factor is the heterogeneity between states of illness and relative effect sizes on inflammatory markers, an example being the fact most COVID-19 patients were tracked only after developing acute symptoms while adjunctive interventions sometimes tracked otherwise healthy patients. In an attempt to detect broader patterns the individual results for each catalyst were combined and averaged. Figure 3 represents averaged SMD for each catalyst, a more detailed set of metrics is available in Appendix A. ${ }^{39,48,53-76}$

\section{COVID-19 Effects}

As expected for a systemic disease, Table 3 indicates COVID-19 exerted significant dysregulating effects across a wide variety of markers. ${ }^{39,48,53-76}$

COVID-19's effects were mostly very large, including major dysregulating effects on CD19, CD4, CD8, C-reactive 


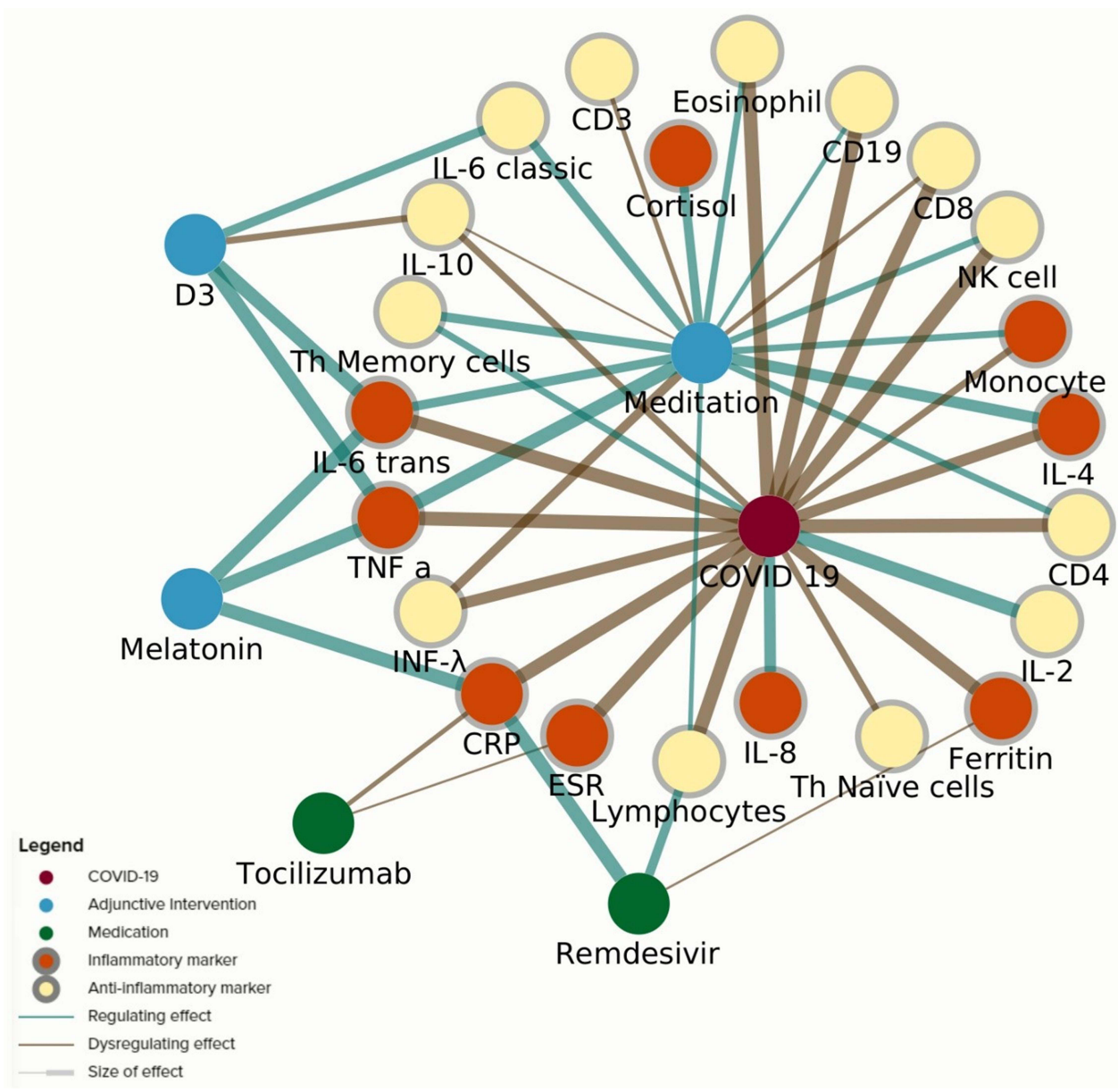

Figure 3 Data point map of catalysts, inflammatory markers, and effect size of connection. Color of the circles identifies whether an inflammatory marker, anti-inflammatory marker, adjunct intervention, medication, or COVID-19 is being referenced. Blue connecting lines indicate a regulating effect from the catalyst to the marker. Brown connecting lines indicate a regulating effect from the catalyst to the marker. The thickness of the connecting line represents the relative effect size of the catalyst on the marker.

protein, eosinophils, erythrocyte sedimentation rate, ferritin, IL-6 trans, overall lymphocytes, natural killer cells, and TNF-a. There was a very large regulating effect on IL-2, which may be a correlation with the body's heightened attempts to curb the runaway inflammation. There were large dysregulating effects on IL-4 and INF- $\lambda$, with a large regulating effect on IL-8. COVID-19 had small dysregulating effects on IL-10, monocyte levels, and Th naïve cells, with a small improvement in Th memory cells.

\section{Pharmacological Effects}

Despite a large body of studies examining Remdesivir and Tocilizumab, few incorporated sufficient data to calculate effect size on specific markers. Where greater detail was available methodology and differentiation of interventions are listed in Appendix A.

\section{Remdesivir}

The data that were available in Table 4 indicated Remdesivir had a very large effect at regulating levels of C-reactive protein, moderate effects at increasing lymphocyte levels, and a negligible dysregulating effect on ferritin levels. ${ }^{39,48,53-76}$

\section{Tocilizumab}

No significant improvements were detected in the averaged SMD for Tocilizumab, as shown in Table 5. ${ }^{39,48,53-76}$ Tocilizumab showed a very small dysregulating effect on C-reactive protein and a negligible dysregulating effect on erythrocyte sedimentation rates.

\section{Adjunctive Intervention Effects}

Where greater detail was available methodology and differentiation of interventions are listed in Appendix A. 
Table 3 Effects of COVID-I9 on Markers, Whether the Effect Was Presumed Regulating or Dysregulating, and the Size of the Effect Using Modified Cohen's d. Differentiation by Severity and/or Time Period of Observation Detailed in Appendix A

\begin{tabular}{|c|c|c|c|c|}
\hline Catalyst & Marker & Impact & Effect Size & SMD \\
\hline COVID 19 & CDI9 & Dysregulating & Very large & -2.04303 \\
\hline COVID 19 & CD4 & Dysregulating & Very large & $-|.564| 4$ \\
\hline COVID 19 & CD8 & Dysregulating & Very large & -2.13114 \\
\hline COVID 19 & CRP & Dysregulating & Very large & 3.98979 I \\
\hline COVID 19 & Eosinophil & Dysregulating & Very large & -4.12104 \\
\hline COVID 19 & ESR & Dysregulating & Very large & 3.153453 \\
\hline COVID 19 & Ferritin & Dysregulating & Very large & 2.691077 \\
\hline COVID 19 & IL-2 & Regulating & Very large & 2.199888 \\
\hline COVID 19 & IL-6 trans & Dysregulating & Very large & 2.820931 \\
\hline COVID 19 & Lymphocytes & Dysregulating & Very large & -2.88716 \\
\hline COVID 19 & NK cell & Dysregulating & Very large & -2.87907 \\
\hline COVID 19 & TNF a & Dysregulating & Very large & 2.424624 \\
\hline COVID 19 & IL-4 & Dysregulating & Large & 0.964237 \\
\hline COVID 19 & IL-8 & Regulating & Large & -0.92577 \\
\hline COVID 19 & INF- $\lambda$ & Dysregulating & Large & -0.98216 \\
\hline COVID 19 & IL-10 & Dysregulating & Small & -0.45513 \\
\hline COVID 19 & Monocyte & Dysregulating & Small & 0.440168 \\
\hline COVID 19 & Th Memory cells & Regulating & Small & 0.32566 \\
\hline COVID 19 & Th Naïve cells & Dysregulating & Small & -0.2364 \\
\hline
\end{tabular}

Table 4 Effects of Remdesivir on Markers, Whether the Effect Was Presumed Regulating or Dysregulating, and the Size of the Effect Using Modified Cohen's d. Differentiation by Severity and/ or Time Period of Observation Detailed in Appendix A

\begin{tabular}{|l|l|l|l|l|}
\hline Catalyst & Marker & Impact & Effect Size & SMD \\
\hline Remdesivir & CRP & Regulating & Very large & -3.34148 \\
Remdesivir & Lymphocytes & Regulating & Medium & 0.727273 \\
Remdesivir & Ferritin & Dysregulating & Negligible & 0.010854 \\
\hline
\end{tabular}

Table 5 Effects of Tocilizumab on Markers, Whether the Effect Was Presumed Regulating or Dysregulating, and the Size of the Effect Using Modified Cohen's d. Differentiation by Severity and/ or Time Period of Observation Detailed in Appendix A

\begin{tabular}{|l|l|l|l|l|}
\hline Catalyst & Marker & Impact & Effect Size & SMD \\
\hline Tocilizumab & CRP & Dysregulating & Very small & 0.101553 \\
Tocilizumab & ESR & Dysregulating & Negligible & 0.018939 \\
\hline
\end{tabular}

Table 6 Effects of Vitamin $D_{3}$ on Markers, Whether the Effect Was Presumed Regulating or Dysregulating, and the Size of the Effect Using Modified Cohen's d. Differentiation by Severity and/ or Time Period of Observation Detailed in Appendix A

\begin{tabular}{|l|l|l|l|l|}
\hline Catalyst & Marker & Impact & Effect Size & SMD \\
\hline D3 & IL-6 trans & Regulating & Very large & -1.65169 \\
D3 & TNF a & Regulating & Very large & -2.1139 \\
D3 & IL-6 classic & Regulating & Medium & 0.772109 \\
D3 & IL-10 & Dysregulating & Small & -0.39462 \\
\hline
\end{tabular}

\section{Vitamin $D_{3}$}

In Table 6 Vitamin $\mathrm{D}_{3}$ demonstrated very large reductions in TNF-a and IL-6 trans levels, moderate effects on increasing IL-6 classic levels, and small dysregulation of IL- $10 .^{39,48,53-76}$

\section{Meditation}

Table 7 indicates that meditation affected a very wide variety of markers, more than any other catalyst besides COVID-19. ${ }^{39,48,53-76}$ Meditation demonstrated very large regulating effects in TNF-a and large regulating effects in IL-4. Moderate regulating effects were seen in cortisol, IL-6 trans (decreased), IL-6 classic (increased), and Th memory cells, with moderate dysregulation to INF- $\lambda$. There were small regulating effects in CD4, eosinophils, monocytes, and natural killer cells. Meditation showed very small regulating effects in CD19 and overall lymphocytes, with a very small dysregulating effect on CD3 and CD8. There was a negligible dysregulating effect on IL- 10 .

It is notable that the research indicating there is both a pro-inflammatory and anti-inflammatory form of IL-6 suggests they would be inversely correlated. ${ }^{25}$ Mean meditation effect sizes show an almost exact inverse correlation between positive and negative effects on IL-6 (0.58 v. -0.57$)$, lending evidence to this premise. 
Table 7 Effects of Meditation on Markers, Whether the Effect Was Presumed Regulating or Dysregulating, and the Size of the Effect Using Modified Cohen's d. Included Studies Focused Primarily on Mindfulness Meditation. Differentiation by Severity and/or Time Period of Observation Detailed in Appendix A

\begin{tabular}{|c|c|c|c|c|}
\hline Catalyst & Marker & Impact & Effect Size & SMD \\
\hline Meditation & TNF a & Regulating & Very large & -1.01499 \\
\hline Meditation & IL-4 & Regulating & Large & -0.8638 \\
\hline Meditation & Cortisol & Regulating & Medium & -0.74358 \\
\hline Meditation & IL-6 classic & Regulating & Medium & 0.58209 \\
\hline Meditation & IL-6 trans & Regulating & Medium & -0.57073 \\
\hline Meditation & INF- $\lambda$ & Dysregulating & Medium & -0.715 \\
\hline Meditation & Th Memory cells & Regulating & Medium & 0.561265 \\
\hline Meditation & CD4 & Regulating & Small & 0.3826 \\
\hline Meditation & Eosinophil & Regulating & Small & 0.31 \\
\hline Meditation & Monocyte & Regulating & Small & -0.293 \\
\hline Meditation & NK cell & Regulating & Small & 0.472 \\
\hline Meditation & CDI9 & Regulating & Very small & 0.149333 \\
\hline Meditation & CD3 & Dysregulating & Very small & -0.11597 \\
\hline Meditation & CD8 & Dysregulating & Very small & -0.17333 \\
\hline Meditation & Lymphocytes & Regulating & Very small & 0.151667 \\
\hline Meditation & IL-10 & Dysregulating & Negligible & -0.095 \\
\hline
\end{tabular}

Table 8 Effects of Melatonin on Markers, Whether the Effect Was Presumed Regulating or Dysregulating, and the Size of the Effect Using Modified Cohen's d. Differentiation by Severity and/ or Time Period of Observation Detailed in Appendix A

\begin{tabular}{|l|l|l|l|l|}
\hline Catalyst & Marker & Impact & Effect Size & SMD \\
\hline Melatonin & CRP & Regulating & Very large & -1.815 \\
Melatonin & IL-6 trans & Regulating & Very large & -2.05 \\
Melatonin & TNF a & Regulating & Very large & -1.89 \\
\hline
\end{tabular}

\section{Melatonin}

As seen in Table 8, melatonin did not affect as many markers as meditation, but it had the greatest number of very large effects of any intervention or medication, with major improvements in C-reactive protein, IL-6 trans, and TNF-a. ${ }^{39,48,53-76}$

\section{Analysis \\ Effect Size Comparison}

Comparisons were examined where two or more catalysts affected a given marker. The markers tracked are grouped into the broad categories of leukocytes and cytokines, with a third category including only one marker, erythrocyte sedimentation rate.

\section{Leukocytes}

As expected, COVID-19 had significantly larger dysregulating effects than any intervention on leukocytes, illustrated in Figure 4. ${ }^{39,48,53-76}$ A surprising finding was the wide variety of markers improved by meditation.

- Meditation had opposite effects to COVID-19 in five out of eight leukocyte comparisons tracked, including CD19, CD4, eosinophils, overall lymphocytes, monocytes, and natural killer cells.

- CD8: There was a very small increase in CD8 with meditation, approximately $8.1 \%$ the size of the COVID-19 effect on CD8.

- Th memory cells: A notable effect was a significantly greater effect by meditation on the production of Th memory cells than was stimulated by introduction of the virus alone. This finding has implications for antiviral strategies.

- Lymphocytes: Remdesivir could only be tracked to overall lymphocytes, but in this instance outperformed meditation in effects contrary to COVID-19. This suggests efficacy for improving sustainable lymphocyte production, though the systemic effects are difficult to track.

\section{Cytokines/Chemokines}

Figure 5 demonstrates that though COVID-19 had significantly greater effects on leukocytes than any of the interventions, influences on cytokines were more 

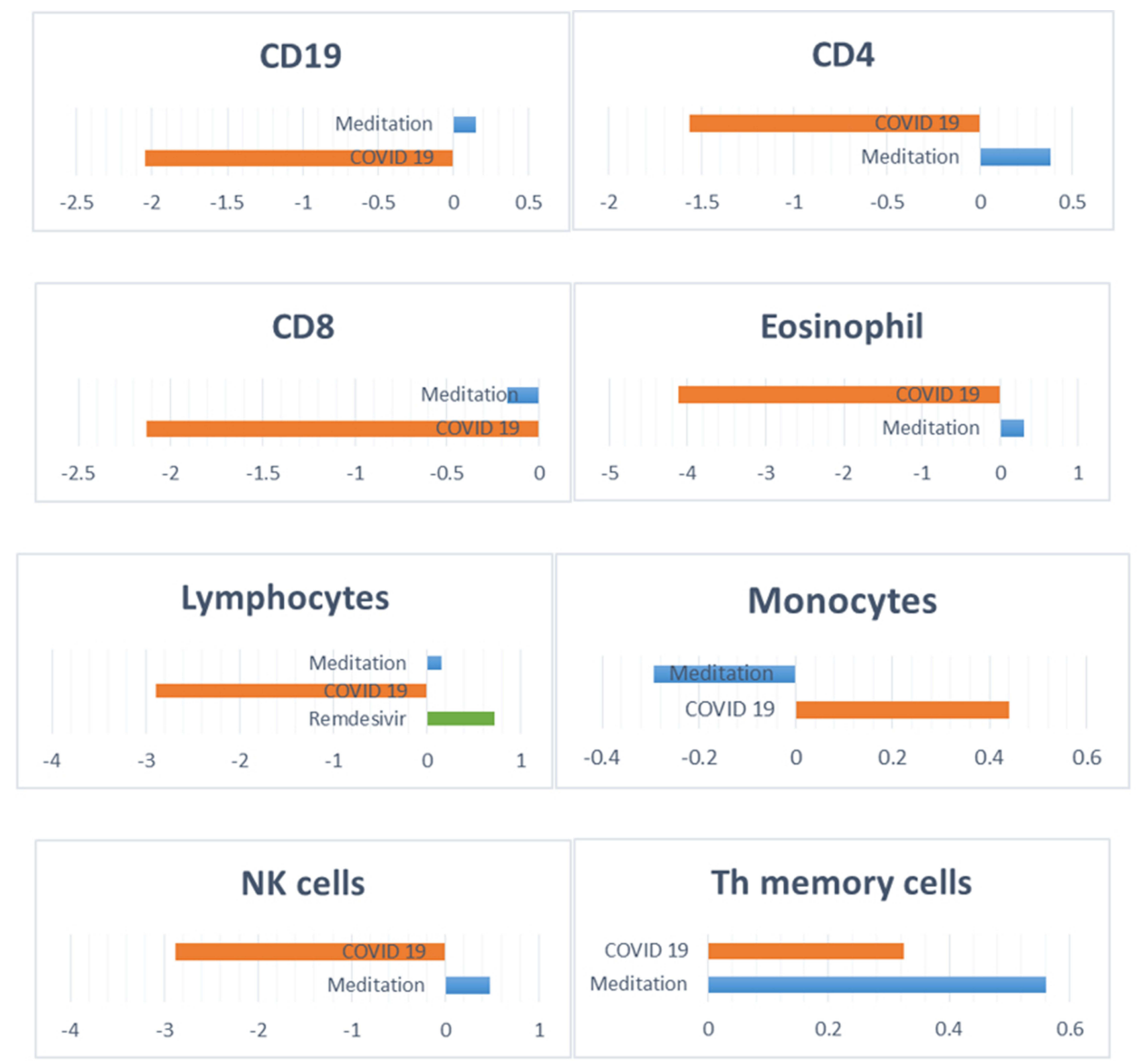

Figure 4 Comparative effects on leukocytes by COVID-19, medication, and adjunct interventions. Horizontal bars track the effects of interventions or COVID-I9, the $\mathrm{X}$-axis indicates the effect size via standard mean difference (SMD). Tracked markers include CDI9, CD4 (Th helper cells), CD8, eosinophils, lymphocytes, monocytes, NK cells (natural killer cells), and TH memory cells.

competitive. ${ }^{39,48,53-76}$ There were also more data for cytokine effects, allowing more comprehensive comparisons.

- C-reactive protein: This marker was tracked across four catalysts, Tocilizumab, Remdesivir, melatonin, and COVID-19. Tocilizumab had a very small dysregulating effect, but both Remdesivir and melatonin showed very large effects in opposition to COVID-19's influence, Remdesivir showing the largest single effect of any intervention on CRP.
- Ferritin: Remdesivir had a negligible effect on ferritin.

- IL-4: Meditation had an opposing effect to COVID19 on IL-4 of an equivalent amount, both having large effect sizes.

- IL-6 classic: Both meditation and vitamin $\mathrm{D}_{3}$ had a medium effect on IL-6 classic, the potentially regulating version of the IL- 6 cytokine. It is notable that meditation and vitamin $D_{3}$ both had effects that increased and decreased IL-6 at approximately 

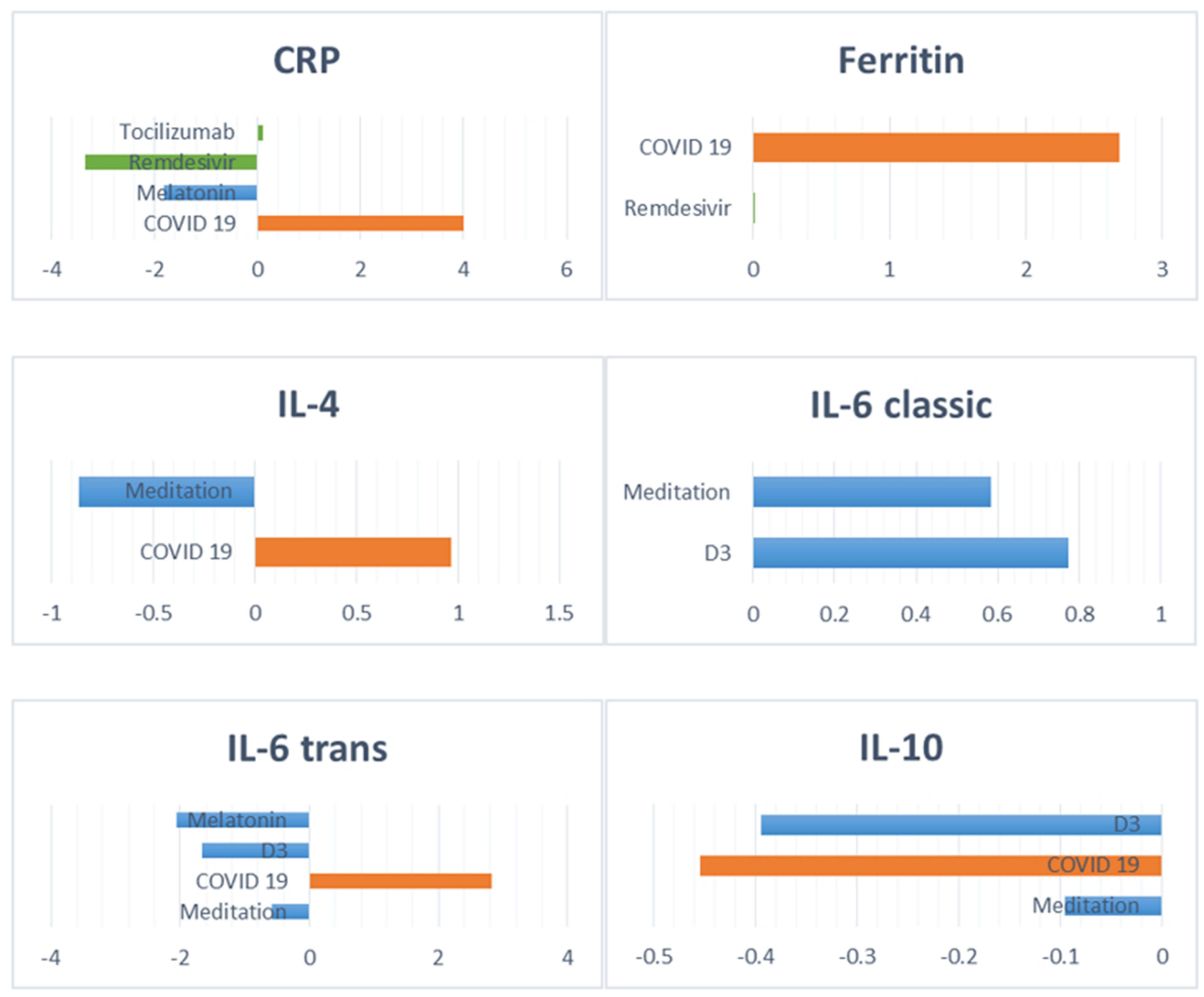

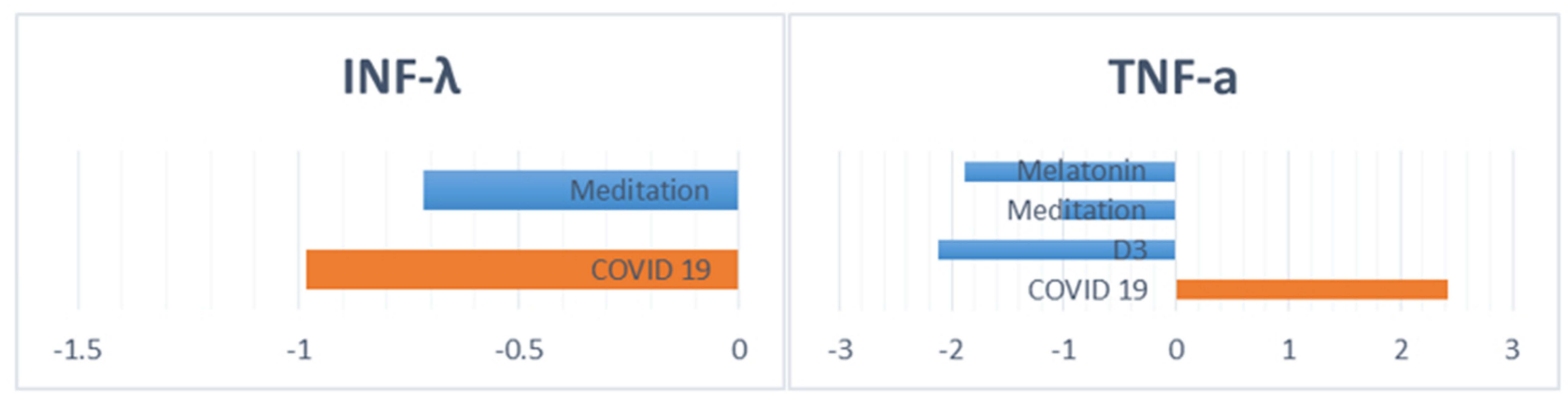

Figure 5 Comparative effects on cytokines by COVID-19, medication, and adjunct interventions. Horizontal bars track the effects of interventions or COVID-19, the X-axis indicates the effect size via standard mean difference (SMD). Tracked markers include CRP (C-reactive protein), ferritin, IL-4 (interleukin 4), IL-6 classic (regulatory interleukin 6), IL-6 trans (dysregulated interleukin 6), IL-20 (interleukin I0), INF $\lambda$ (interferons $\lambda$ ), and TNF-a (tumor necrosis factor alpha).

inverse amounts depending on the study, lending further evidence to the concept of a trans and classic form of IL-6.

- IL-6 trans: As the most prominent indicator of cytokine storms, IL-6 trans was tracked by multiple catalysts, though insufficient data were found for Remdesivir and Tocilizumab. Melatonin and vitamin $\mathrm{D}_{3}$ both showed a very large effect on IL-6 trans, though still somewhat smaller than the opposing effect of COVID-19. Meditation showed a medium 
sized effect on IL-6 trans. It is notable that meditation and vitamin $\mathrm{D}_{3}$ both had effects that increased and decreased IL- 6 at approximately inverse amounts depending on the study, lending further evidence to the concept of a trans and classic form of IL-6.

- IL-10: Provided unexpected results, with COVID-19, vitamin $\mathrm{D}_{3}$, and meditation all increasing the marker to some degree, meditation to a significantly smaller degree.

- INF- $\lambda$ : Meditation had a medium sized effect on INF$\lambda$ that corresponded with a large effect by COVID19. The complex relationship of interferons with various immune functions makes this correlation difficult to analyze.

- Tumor necrosis alpha: Along with IL-6, TNF-a is a cytokine frequently linked to dysregulated inflammation. Melatonin, vitamin $\mathrm{D}_{3}$, and meditation all showed a very large effect in opposition to COVID19 's very large effect.

\section{ESR}

Figure 6 tracks erythrocyte sedimentation rate, a common hematological test to measure levels of inflammation. ${ }^{39,48,53-76}$

- ESR: Tocilizumab showed a negligible effect on ESR in comparison to COVID-19's very large effect.

\section{System Connections}

Meaningful system analysis requires an understanding of the connections between parts of the system. This work is not intended to conduct such an analysis but can provide information on the connections to be used in future research. These simple network metrics reflect the number and direction of connections compiled in this research.

\section{ESR}

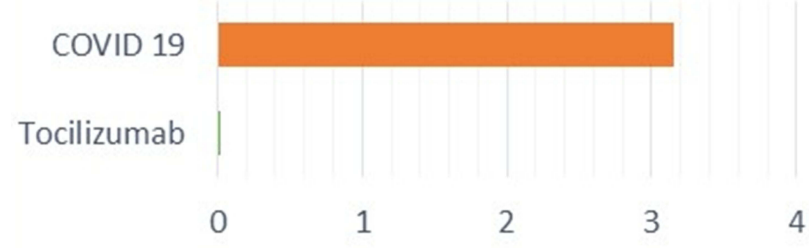

Figure 6 Comparative effects on ESR (erythrocyte sedimentation rate) by COVID19, medication, and adjunct interventions.

\section{Degree Centrality}

The simplest of network metrics, these numbers represent the total number of connections of any kind that belong to a data point. Degree centrality is useful for determining hubs of interaction but cannot determine direction of influence.

\section{Indegree}

Unlike degree centrality, indegree measures directional connections, specifically the data points receiving incoming connections. Given the focus of this research, indegree is primarily made up of inflammatory markers. Higher values reflect a greater degree of influence from the rest of the system.

\section{Outdegree}

Whereas indegree measures incoming connections, outdegree tracks the number of outgoing connections from a given data point. Given the focus of this research, indegree is made up of catalysts. Higher values reflect a greater degree of influence upon the rest of the system. These connections were calculated in Table 9 based on the relationships discovered in the analyzed body of research. $^{39,48,53-76}$

An examination of the degrees of connection revealed COVID-19 and meditation were by far the most widely influential catalysts. The markers most affected by any catalyst included IL-6 trans, TNF-a, and CRP, followed by IL-10 and overall lymphocytes.

\section{Discussion}

The initial hypothesis asks whether adjunct interventions show any significant effects on inflammatory markers that are dysregulated by COVID-19. These effects were compared to Remdesivir and Tocilizumab, some of the leading SARS-CoV2 medications, to determine whether there are any unique benefits. The findings demonstrate that adjunct interventions not only affect a greater number of markers than Remdesivir or Tocilizumab, the effect sizes of adjunct treatments are often as large or larger. Melatonin, vitamin $\mathrm{D}_{3}$, and meditation affected 23 inflammatory markers while Remdesivir and Tocilizumab affected 5. Adjunctive interventions affected $68.4 \%$ of the inflammatory markers impacted by COVID-19, standard pharmaceutical medication affected $26.3 \%$ of the inflammatory markers impacted by COVID-19. Melatonin, vitamin $\mathrm{D}_{3}$, and meditation had very large or large effects on 7 inflammatory markers, Remdesivir and Tocilizumab had very large or large effects on 1 inflammatory marker, though that single effect was extremely large. 
Table 9 Top Twenty Data Points and Values of the Network Metrics, Tracking Degree Centrality, Indegree, and Outdegree

\begin{tabular}{|c|c|c|c|c|c|}
\hline Degree Centrality & Value & Indegree & Value & Outdegree & Value \\
\hline COVID 19 & 19 & IL-6 trans & 4 & COVID 19 & 19 \\
\hline Meditation & 16 & TNF a & 4 & Meditation & 16 \\
\hline D3 & 4 & CRP & 4 & D3 & 4 \\
\hline IL-6 trans & 4 & IL-10 & 3 & Melatonin & 3 \\
\hline TNF a & 4 & Lymphocytes & 3 & Remdesivir & 3 \\
\hline CRP & 4 & CD8 & 2 & Tocilizumab & 2 \\
\hline Melatonin & 3 & IL-4 & 2 & CD8 & 0 \\
\hline IL-10 & 3 & CDI9 & 2 & Cortisol & 0 \\
\hline Remdesivir & 3 & ESR & 2 & IL-2 & 0 \\
\hline Lymphocytes & 3 & IL-6 classic & 2 & IL-6 trans & 0 \\
\hline CD8 & 2 & Eosinophil & 2 & IL-4 & 0 \\
\hline IL-4 & 2 & INF- $\lambda$ & 2 & CDI9 & 0 \\
\hline CDI9 & 2 & CD4 & 2 & IL-10 & 0 \\
\hline ESR & 2 & NK cell & 2 & IL-8 & 0 \\
\hline IL-6 classic & 2 & Th Memory cells & 2 & ESR & 0 \\
\hline Eosinophil & 2 & Monocyte & 2 & TNF a & 0 \\
\hline INF- $\lambda$ & 2 & Ferritin & 2 & IL-6 classic & 0 \\
\hline CD4 & 2 & Cortisol & I & Eosinophil & 0 \\
\hline NK cell & 2 & IL-2 & I & Lymphocytes & 0 \\
\hline Th memory cells & 2 & IL-8 & I & CRP & 0 \\
\hline
\end{tabular}

Though the impacts of Tocilizumab are clearly smaller in both effect size and number of markers affected, comparisons between Remdesivir, melatonin, vitamin $\mathrm{D}_{3}$, and meditation are significantly more nuanced. In comparison to Remdesivir the adjunctive treatments affected a greater quantity of markers, with a far greater number of large effects, yet Remdesivir exhibited a uniquely large effect size on C-reactive proteins. Uneven distribution of both effect sizes and markers affected suggests different mechanisms operating behind the adjunctive treatments and Remdesivir. In this scenario the adjunct treatments and Remdesivir might operate synergistically, influencing different inflammatory pathways via different mechanisms.

Melatonin, vitamin $\mathrm{D}_{3}$, and meditation should be considered for treatment of COVID-19 alongside standards of care. Though the precise interactions between markers and disease outcomes are unclear, evidence suggests these interventions have significant, quantifiable effects on the inflammatory markers associated with COVID-19's dysregulation.

\section{Implications}

There are short and long-term implications behind the evidence supporting the efficacy of adjunct interventions to improve inflammatory dysregulation. In the immediate future these adjunct interventions could be considered for use alongside standard medication. The cost-efficiency and lack of serious side effects or contraindications associated with reasonable applications of meditation, vitamin $D_{3}$, and melatonin makes for a simple, affordable, and easy combination with most treatment plans. The increased scope of regulating systemic inflammation could result in significantly lower rates of cytokine storms, potentially saving many lives.

The long-term benefit to this approach lies in the focus on systemic dysregulation rather than acute events. The current healthcare system is highly vulnerable to the ripple effects of inflammatory viruses on chronically ill patients, posing high individual and institutional risk. COVID-19 is particularly systemic in its pathology, yet there are many communicable and non-communicable diseases that are heavily influenced by interconnected, dysregulated inflammation. A greater focus on systemic diseases and interventions is highlighted in this pandemic, but advances in this field could have lasting benefits across numerous fields of medicine. It is likely there is no single solution to either the pandemic or these institutional weaknesses, but rather a combination of interventions and policies are necessary to stimulate the entire organism to adapt.

Further research, both into the adjunct interventions listed here and into system-wide interventions overall, should be considered warranted and pressing. 


\section{Limitations}

This research has several conditions and limits that must be considered. The act of aggregating, calculating, approximating, and averaging effect sizes from very disparate sources creates the possibility of imprecision at multiple levels. As referenced above, differences in the initial health conditions and observation intervals of the participants in these studies are complicating factors that warrant examination. The consistent findings of data from distinct sources lends credibility to the overall findings, but review of the differentiated data is available and encouraged in Appendix A. The selection, characterization, and contextualization of highly complex inflammatory markers is likewise inherently approximate. Limited data on markers associated with many interventions also prevented a wider comparative analysis among standard medical approaches. In particular, it is important to note that although insufficient data were found to calculate effect sizes on IL- 6 by Tocilizumab, this intervention is primarily known as an IL-6 inhibitor, so it can be assumed it has a significant effect on that marker. ${ }^{82}$

While Tocilizumab and Remdesivir were being studied as leading interventions when this study began, by the time of publication they have been largely supplanted by more effective treatments. Comparison with contemporary standards of care is warranted. Most COVID-19 effects were tracked after admission to a hospital or care center, creating statistical sampling issues in relation to asymptomatic or misdiagnosed infections. Long-term examination of changes in inflammatory loads among asymptomatic infections is necessary to expand marker effect size accuracy.

Several emergent Phase II/III studies on artemisinin and derivatives have released preliminary data suggesting a reduced time of conversion to negativity and reduction in severe symptoms. ${ }^{83}$ Artemisia and its bioactive constituents represent promising examples of adjunct therapeutics for broad spectrum antiviral effects, reduction in tissue fibrosis, and inhibition of key cytokines such as TGF-beta, benefits that have been well established in malaria treatments. However, the artemisinin studies did not evaluate serum cytokines and could not be included in the current meta-analysis; thus future clinical trials administering these compounds and others should extensively evaluate serum biomarkers.

Finally, a critical limitation is a lack of understanding between the mechanisms of inflammatory markers and the epidemiological behavior of COVID-19. This paper explores effects upon inflammatory markers associated with COVID-19, but the complex dynamics of those markers cannot yet be used to predict differences in treatment outcomes. Further exploration of the threshold for cytokine storms is necessary before any precise benefits in reducing individual markers can be determined.

\section{Disclosure}

The authors report no conflicts of interest for this work.

\section{References}

1. Pollard CA, Morran MP, Nestor-Kalinoski AL. The COVID-19 pandemic: a global health crisis. Physiol Genomics. 2020;52 (11):549-557. doi:10.1152/physiolgenomics.00089.2020

2. Singh AK, Gillies CL, Singh R, et al. Prevalence of co-morbidities and their association with mortality in patients with COVID-19: a systematic review and meta-analysis. Diabetes Obes Metab. 2020;22(10):1915-1924. doi:10.1111/dom.14124

3. Gustine JN, Jones D. Immunopathology of hyperinflammation in COVID-19. Am J Pathol. 2021;191(1):4-17. doi:10.1016/j. ajpath.2020.08.009

4. Panigrahy D, Gilligan MM, Huang S, et al. Inflammation resolution: a dual-pronged approach to averting cytokine storms in COVID-19? Cancer Metastasis Rev. 2020;39(2):337-340. doi:10.1007/s10555020-09889-4

5. Zabetakis I, Lordan R, Norton C, Tsoupras A. COVID-19: the inflammation link and the role of nutrition in potential mitigation. Nutrients. 2020;12:5. doi:10.3390/nu12051466

6. Shakoor H, Feehan J, Al Dhaheri AS, et al. Immune-boosting role of vitamins D, C, E, zinc, selenium and omega-3 fatty acids: could they help against COVID-19? Maturitas. 2021;143:1-9. doi:10.1016/j. maturitas.2020.08.003

7. Hunter P. The inflammation theory of disease. The growing realization that chronic inflammation is crucial in many diseases opens new avenues for treatment. EMBO Rep. 2012;13(11):968-970. doi:10.1038/embor.2012.142

8. Rea IM, Gibson DS, McGilligan V, McNerlan SE, Alexander HD, Ross OA. Age and age-related diseases: role of inflammation triggers and cytokines. Front Immunol. 2018;9:586. doi:10.3389/ fimmu.2018.00586

9. Libby P. Inflammatory mechanisms: the molecular basis of inflammation and disease. Nutr Rev. 2007;65(12 Pt 2):S140-S146. doi:10.1111/j.1753-4887.2007.tb00352.x

10. Conti P, Ronconi G, Caraffa A, et al. Induction of pro-inflammatory cytokines (IL-1 and IL-6) and lung inflammation by coronavirus-19 (COVI-19 or SARS-CoV-2): anti-inflammatory strategies. J Biol Regul Homeost Agents. 2020;34:2. doi:10.23812/CONTI-E

11. Amiri-Dashatan N, Koushki M, Ghorbani F, Naderi N. Increased inflammatory markers correlate with liver damage and predict severe COVID-19: a systematic review and meta-analysis. Gastroenterol Hepatol Bed Bench. 2020;13(4):282-291.

12. Renu K, Prasanna PL, Valsala Gopalakrishnan A. Coronaviruses pathogenesis, comorbidities and multi-organ damage - A review. Life Sci. 2020;255:117839. doi:10.1016/j.lfs.2020.117839

13. Black PH. Stress and the inflammatory response: a review of neurogenic inflammation. Brain Behav Immun. 2002;16(6):622-653. doi:10.1016/s0889-1591(02)00021-1

14. Coronavirus Disease (COVID-19) situation reports. Avaiable from: https://www.who.int/emergencies/diseases/novel-coronavirus-2019/ situation-reports. Accessed January 28, 2021.

15. Wang F, Kream RM, Stefano GB. Long-term respiratory and neurological sequelae of COVID-19. Med Sci Monit. 2020;26:e928996. doi:10.12659/MSM.928996 
16. Verkhratsky A, Li Q, Melino S, Melino G, Shi Y. Can COVID-19 pandemic boost the epidemic of neurodegenerative diseases? Biol Direct. 2020;15(1):28. doi:10.1186/s13062-020-00282-3

17. McDonald LT. Healing after COVID-19: are survivors at risk for pulmonary fibrosis? Am J Physiol Lung Cell Mol Physiol. 2021;320 (2):L257-L265. doi:10.1152/ajplung.00238.2020

18. O'Sullivan O. Long-term sequelae following previous coronavirus epidemics. Clin Med. 2021;21(1):e68-e70. doi:10.7861/ clinmed.2020-0204

19. Saklayen MG. The global epidemic of the metabolic syndrome. Curr Hypertens Rep. 2018;20:2. doi:10.1007/s11906-018-0812-z

20. Leijte WT, Wagemaker NMM, van Kraaij TDA, et al. Mortality and re-admission after hospitalization with COVID-19. Ned Tijdschr Geneeskd. 2020;164:D5423.

21. NIH launches new initiative to study. "Long COVID." National Institutes of Health (NIH); February 23, 2021. Available from: https:// www.nih.gov/about-nih/who-we-are/nih-director/statements/nihlaunches-new-initiative-study-long-covid. Accessed March 30, 2021.

22. Li S, Zhang Y, Guan Z, et al. SARS-CoV-2 triggers inflammatory responses and cell death through caspase-8 activation. Signal Transduct Target Ther. 2020;5(1):235. doi:10.1038/s41392-020-00334-0

23. Pum A, Ennemoser M, Adage T, Kungl AJ. Cytokines and chemokines in SARS-CoV-2 infections-therapeutic strategies targeting cytokine storm. Biomolecules. 2021;11:1. doi:10.3390/biom11010091

24. Choudhary S, Sharma K, Silakari O. The interplay between inflammatory pathways and COVID-19: a critical review on pathogenesis and therapeutic options. Microb Pathog. 2021;150:104673. doi:10.1016/j.micpath.2020.104673

25. Chen Y-M, Zheng Y, Yu Y, et al. Blood molecular markers associated with COVID-19 immunopathology and multi-organ damage. EMBO J. 2020;39(24):e105896. doi:10.15252/ embj.2020105896

26. Mak JC, Chan-Yeung MM, Ho SP, et al. Elevated plasma TGF-beta1 levels in patients with chronic obstructive pulmonary disease. Respir Med. 2009;103:1083-1089. doi:10.1016/j.rmed.2009.01.005

27. Xiong Y, Liu Y, Cao L, et al. Transcriptomic characteristics of bronchoalveolar lavage fluid and peripheral blood mononuclear cells in COVID-19 patients. Emerg Microbes Infect. 2020;9:761-770. doi:10.1080/22221751.2020.1747363

28. Woods PS, Tazi MF, Chesarino NM, Amer AO, Davis IC. TGF-betainduced IL-6 prevents development of acute lung injury in influenza A virus-infected F508del CFTR-heterozygous mice. Am J Physiol Lung Cell Mol Physiol. 2015;308:L1136-L1144. doi:10.1152/ ajplung.00078.2015

29. Mu E, Ding R, An X, Li X, Chen S, Ma X. Heparin attenuates lipopolysaccharide-induced acute lung injury by inhibiting nitric oxide synthase and TGF-beta/Smad signaling pathway. Thromb Res. 2012;129:479-485. doi:10.1016/j.thromres.2011.10.003

30. Pittet JF, Griffiths MJ, Geiser T, et al. TGF-beta is a critical mediator of acute lung injury. J Clin Invest. 2001;107:1537-1544. doi:10.1172/ JCI11963

31. Chen W. A potential treatment of COVID-19 with TGF-beta blockade. Int J Biol Sci. 2020;16:1954-1955. doi:10.7150/ijbs.46891

32. Hu X, Huang X. Alleviation of inflammatory response of pulmonary fibrosis in acute respiratory distress syndrome by puerarin via Transforming Growth Factor (TGF-beta1). Med Sci Monit. 2019;25:6523-6531. doi:10.12659/MSM.915570

33. Li SW, Wang CY, Jou YJ, et al. SARS coronavirus papain-like protease induces Egr-1-dependent up-regulation of TGF-beta1 via ROS/p38 MAPK/STAT3 pathway. Sci Rep. 2016;6:25754. doi:10.1038/srep25754

34. Wang CY, Lu CY, Li SW, et al. SARS coronavirus papain-like protease up-regulates the collagen expression through non-Samd TGF-beta1 signaling. Virus Res. 2017;235:58-66. doi:10.1016/j. virusres.2017.04.008
35. Mendelson M, Nel J, Blumberg L, et al. Long-COVID: an evolving problem with an extensive impact. $S$ Afr Med J. 2020;111(1):10-12. doi:10.7196/SAMJ.2020.v111i11.15433

36. Ali A, Katz DL. Disease prevention and health promotion. Am J Prev Med. 2015;49(50 3):S230-S240. doi:10.1016/j.amepre.2015.07.019

37. Morgan N, Irwin MR, Chung M, Wang C, Bacurau RFP. The effects of mind-body therapies on the immune system: meta-analysis. PLoS One. 2014;9(7):e100903. doi:10.1371/journal.pone.0100903

38. Jolliffe DA, Camargo CA, Sluyter JD, et al. Vitamin D supplementation to prevent acute respiratory infections: a systematic review and meta-analysis of aggregate data from randomised controlled trials. Lancet Diabetes Endocrinol. 2021;9 (5):276-292. doi:10.1016/S2213-8587(21)00051-6

39. Zhang R, Wang X, Ni L, et al. COVID-19: melatonin as a potential adjuvant treatment. Life Sci. 2020;250:117583. doi:10.1016/j. lfs. 2020.117583

40. Zerr P, Vollath S, Palumbo-Zerr K, et al. Vitamin D receptor regulates TGF-beta signalling in systemic sclerosis. Ann Rheum Dis. 2015;74: e20. doi:10.1136/annrheumdis-2013-204378

41. Isik S, Ozuguz U, Tutuncu YA, et al. Serum transforming growth factor-beta levels in patients with vitamin D deficiency. Eur J Intern Med. 2012;23:93-97. doi:10.1016/j.ejim.2011.09.017

42. Kapepula PM, Kabengele JK, Kingombe M, et al. Artemisia Spp. derivatives for COVID-19 treatment: anecdotal use, political hype, treatment potential, challenges, and road map to randomized clinical trials. $\mathrm{Am}$ J Trop Med Hyg. 2020;103:960-964. doi:10.4269/ajtmh.20-0820

43. Uckun FM, Saund S, Windlass H, Trieu V. Repurposing anti-malaria phytomedicine artemisinin as a COVID-19 drug. Front Pharmacol. 2021;12:649532. doi:10.3389/fphar.2021.649532

44. Entrenas Castillo M, Entrenas Costa LM, Vaquero Barrios JM, et al. Effect of calcifediol treatment and best available therapy versus best available therapy on intensive care unit admission and mortality among patients hospitalized for COVID-19: a pilot randomized clinical study. J Steroid Biochem Mol Biol. 2020;203:105751. doi:10.1016/j.jsbmb.2020.105751

45. Ling SF, Broad E, Murphy R, et al. High-dose cholecalciferol booster therapy is associated with a reduced risk of mortality in patients with COVID-19: a Cross-Sectional Multi-Centre Observational Study. Nutrients. 2020;12:12. doi:10.3390/nu12123799

46. Grant WB, Lahore H, McDonnell SL, et al. Evidence that vitamin D supplementation could reduce risk of influenza and COVID-19 infections and deaths. Nutrients. 2020;12(4). doi:10.3390/nu12040988

47. Feitosa EL, Júnior FTDSS, Nery Neto JADO, et al. COVID-19: rational discovery of the therapeutic potential of melatonin as a SARS-CoV-2 main protease inhibitor. Int $J$ Med Sci. 2020;17 (14):2133-2146. doi:10.7150/ijms.48053

48. Cahn BR, Goodman MS, Peterson CT, Maturi R, Mills PJ. Yoga, meditation and mind-body health: increased BDNF, cortisol awakening response, and altered inflammatory marker expression after a 3-month yoga and meditation retreat. Front Hum Neurosci. 2017;11:315. doi:10.3389/fnhum.2017.00315

49. Bushell WC, Theise ND. Toward a unified field of study: longevity, regeneration, and protection of health through meditation and related practices. Ann N Y Acad Sci. 2009;1172:5-19. doi:10.1111/j.17496632.2009.04959.x

50. Wang Y, Zhang D, Du G, et al. Remdesivir in adults with severe COVID-19: a randomised, double-blind, placebo-controlled, multicentre trial. Lancet. 2020;395(10236):1569-1578. doi:10.1016/ S0140-6736(20)31022-9

51. Sheppard M, Laskou F, Stapleton PP, Hadavi S, Dasgupta B. Tocilizumab (Actemra). Hum Vaccin Immunother. 2017;13 (9):1972-1988. doi:10.1080/21645515.2017.1316909

52. Bushell W, Castle R, Williams MA, et al. Meditation and yoga practices as potential adjunctive treatment of SARS-CoV-2 infection and COVID-19: a brief overview of key subjects. $J$ Altern Complement Med. 2020;26(7):547-556. doi:10.1089/acm.2020.0177 
53. Aiswarya D, Arumugam V, Dineshkumar T, et al. Use of remdesivir in patients with COVID-19 on hemodialysis: a Study of Safety and Tolerance. Kidney Int Rep. 2021;6(3):586-593. doi:10.1016/j. ekir.2020.12.003

54. Akbari H, Tabrizi R, Lankarani KB, et al. The role of cytokine profile and lymphocyte subsets in the severity of coronavirus disease 2019 (COVID-19): a systematic review and meta-analysis. Life Sci. 2020;258:118167. doi:10.1016/j.1fs.2020.118167

55. Alamdari NM, Mahdavi R, Roshanravan N, et al. Placebo-controlled trial related to the effects of melatonin on oxidative stress and inflammatory parameters of obese women. Horm Metab Res. 2015;47(07):504-508. doi:10.1055/s-0034-1384587

56. Bartels LE, Jørgensen SP, Bendix M, et al. 25-hydroxy vitamin D3 modulates dendritic cell phenotype and function in crohn's disease. Inflammopharmacology. 2013;21(2):177-186. doi:10.1007/s10787012-0168-y

57. Bower JE, Crosswell AD, Stanton AL, et al. Mindfulness meditation for younger breast cancer survivors: a randomized controlled trial. Cancer. 2015;121(8):1231-1240. doi:10.1002/cncr.29194

58. Carlson LE, Speca M, Patel KD, Goodey E. Mindfulness-based stress reduction in relation to quality of life, mood, symptoms of stress, and immune parameters in breast and prostate cancer outpatients. Psychosom Med. 2003;65(4):571-581. doi:10.1097/01.psy.0000074003.35911.41

59. Carlson LE, Speca M, Patel KD, Goodey E. Mindfulness-based stress reduction in relation to quality of life, mood, symptoms of stress and levels of cortisol, dehydroepiandrosterone sulfate (DHEAS) and melatonin in breast and prostate cancer outpatients. Psychoneuroendocrinology. 2004;29(4):448-474. doi:10.1016/s0306-4530(03)00054-4

60. Carlson LE, Speca M, Faris P, Patel KD. One year pre-post intervention follow-up of psychological, immune, endocrine and blood pressure outcomes of mindfulness-based stress reduction (MBSR) in breast and prostate cancer outpatients. Brain Behav Immun. 2007;21 (8):1038-1049. doi:10.1016/j.bbi.2007.04.002

61. Celinski K, Konturek PC, Slomka M, et al. Effects of treatment with melatonin and tryptophan on liver enzymes, parameters of fat metabolism and plasma levels of cytokines in patients with non-alcoholic fatty liver disease-14 months follow up. J Physiol Pharmacol. 2014;65(1):75-82.

62. Chen G, Wu D, Guo W, et al. Clinical and immunological features of severe and moderate coronavirus disease 2019. J Clin Invest. 2020;130(5):2620-2629. doi:10.1172/JCI137244

63. Chen L, Liu HG, Liu W, et al. Analysis of clinical features of 29 patients with 2019 novel coronavirus pneumonia. Zhonghua Jie He He Hu Xi Za Zhi. 2020;43:E005. doi:10.3760/cma.j.issn.10010939.2020.0005

64. Cichoz-Lach H, Celinski K, Konturek PC, Konturek SJ, Slomka M. The effects of L-tryptophan and melatonin on selected biochemical parameters in patients with steatohepatitis. J Physiol Pharmacol. 2010;61(5):577-580.

65. Epel ES, Puterman E, Lin J, et al. Meditation and vacation effects have an impact on disease-associated molecular phenotypes. Transl Psychiatry. 2016;6(8):e880. doi:10.1038/tp.2016.164

66. Gonzalez-Garcia M, Ferrer MJ, Borras X, et al. Effectiveness of mindfulness-based cognitive therapy on the quality of life, emotional status, and CD4 cell count of patients aging with HIV infection. AIDS Behav. 2014;18(4):676-685. doi:10.1007/s10461-013-0612-z

67. Javanmard SH, Heshmat-Ghahdarijani K, Mirmohammad-Sadeghi M, Sonbolestan SA, Ziayi A. The effect of melatonin on endothelial dysfunction in patient undergoing coronary artery bypass grafting surgery. $A d v$ Biomed Res. 2016;5:174. doi:10.4103/2277-9175.194801

68. Lengacher Ca, Kip KE, Post-White J, et al. Lymphocyte recovery after breast cancer treatment and mindfulness-based stress reduction (MBSR) therapy. Biol Res Nurs. 2013;15(1):37-47. doi:10.1177/ 1099800411419245
69. Qin $\mathrm{C}$, Zhou $\mathrm{L}, \mathrm{Hu} \mathrm{Z}$, et al. Dysregulation of immune response in patients with COVID-19 in Wuhan, China. Clin Infect Dis. 2020. doi:10.1093/cid/ciaa248

70. Rausch-Fan X, Leutmezer F, Willheim M, et al. Regulation of cytokine production in human peripheral blood mononuclear cells and allergen-specific th cell clones by 1alpha,25-dihydroxyvitamin D3. Int Arch Allergy Immunol. 2002;128(1):33-41. doi:10.1159/000058001

71. Raygan F, Ostadmohammadi V, Bahmani F, Reiter RJ, Asemi Z. Melatonin administration lowers biomarkers of oxidative stress and cardio-metabolic risk in type 2 diabetic patients with coronary heart disease: a randomized, double-blind, placebo-controlled trial. Clin Nutr. 2019;38(1):191-196. doi:10.1016/j.clnu.2017.12.004

72. Rosenkranz MA, Lutz A, Perlman DM, et al. Reduced stress and inflammatory responsiveness in experienced meditators compared to a matched healthy control group. Psychoneuroendocrinology. 2016;68:117-125. doi:10.1016/j.psyneuen.2016.02.013

73. Shete SU, Verma A, Kulkarni DD, Bhogal RS. Effect of yoga training on inflammatory cytokines and C-reactive protein in employees of small-scale industries. J Educ Health Promot. 2017;6:76. doi:10.4103/jehp.jehp_65_17

74. Smolen JS, Beaulieu A, Rubbert-Roth A, et al. Effect of interleukin-6 receptor inhibition with tocilizumab in patients with rheumatoid arthritis (OPTION study): a double-blind, placebo-controlled, randomised trial. Lancet. 2008;371(9617):987-997. doi:10.1016/S01406736(08)60453-5

75. Wang F, Nie J, Wang H, et al. Characteristics of peripheral lymphocyte subset alteration in COVID-19 pneumonia. J Infect Dis. 2020;221(11):1762-1769. doi:10.1093/infdis/jiaa150

76. Witek-Janusek L, Albuquerque K, Chroniak KR, Chroniak C, Durazo-Arvizu R, Mathews HL. Effect of mindfulness based stress reduction on immune function, quality of life and coping in women newly diagnosed with early stage breast cancer. Brain Behav Immun. 2008;22(6):969-981. doi:10.1016/j.bbi.2008.01.012

77. Meng J, Xiao G, Zhang J, et al. Renin-angiotensin system inhibitors improve the clinical outcomes of COVID-19 patients with hypertension. Emerg Microbes Infect. 2020;9(1):757-760. doi:10.1080/22221751.2020.1746200

78. Ramezani M, Simani L, Karimialavijeh E, Rezaei O, Hajiesmaeili M, Pakdaman H. The role of anxiety and cortisol in outcomes of patients with covid-19. Basic Clin Neurosci. 2020;11(2):179-184. doi:10.32598/bcn.11.covid19.1168.2

79. Magro G. SARS-CoV-2 and COVID-19: is interleukin-6 (IL-6) the 'culprit lesion' of ARDS onset? What is there besides tocilizumab? SGP130Fc. Cytokine $X . \quad 2020 ; 2(2): 100029$. doi:10.1016/j. cytox.2020.100029

80. Wasserstein RL, Lazar NA. The ASA statement on p-values: context, process, and purpose. Am Stat. 2016;70:2129-2133. doi:10.1080/ 00031305.2016.1154108

81. Faraone SV. Interpreting estimates of treatment effects. $P T .2008 ; 33$ (12):700-711.

82. Mihara M 1, Kasutani K, Okazaki M, et al. Tocilizumab inhibits signal transduction mediated by both mIL-6R and sIL-6R, but not by the receptors of other members of IL-6 cytokine family. Int Immunopharmacol. 2005;5(12):1731-1740. doi:10.1016/j.intimp.2005.05.010

83. Lin Y, Wu F, Xie Z, et al. Clinical study of artesunate in the treatment of coronavirus disease 2019. Zhonghua Wei Zhong Bing Ji Jiu Yi Xue. 2020;32:417-420. doi:10.3760/cma.j.cn121430-20200312-00412 
Video abstract

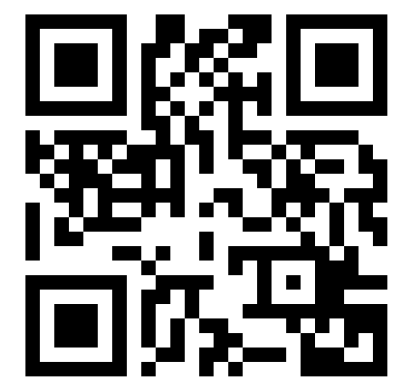

Point your SmartPhone at the code above. If you have a QR code reader the video abstract will appear. Or use: https://youtu.be/2i2QG5ITOJI

\section{Publish your work in this journal}

The Journal of Inflammation Research is an international, peerreviewed open-access journal that welcomes laboratory and clinical findings on the molecular basis, cell biology and pharmacology of inflammation including original research, reviews, symposium reports, hypothesis formation and commentaries on: acute/chronic inflammation; mediators of inflammation; cellular processes; molecular mechanisms; pharmacology and novel anti-inflammatory drugs; clinical conditions involving inflammation. The manuscript management system is completely online and includes a very quick and fair peerreview system. Visit http://www.dovepress.com/testimonials.php to read real quotes from published authors. 